\title{
Prescription practice and clinical utility of chest radiographs in a pediatric intensive care unit: a prospective observational study
}

Rajeev Gupta ${ }^{1}$, Karthi Nallasamy ${ }^{1 *}$, Vijai Williams ${ }^{1}$, Akshay Kumar Saxena ${ }^{2}$ and Muralidharan Jayashree ${ }^{1}$

\begin{abstract}
Background: Chest radiograph (CXR) prescribing pattern and practice vary widely among pediatric intensive care units (PICU). 'On demand' approach is increasingly recommended as against daily'routine' CXRs; however, the realworld practice is largely unknown.
\end{abstract}

Methods: This was a prospective observational study performed in children younger than 12 years admitted to PICU of a tertiary care teaching hospital in India. Data were collected on all consecutive CXRs performed between December 2016 and April 2017. The primary outcome was to assess the factors that were associated with higher chest radiograph prescriptions in PICU. Secondary outcomes were to study the indications, association with mechanical ventilation, image quality and avoidable radiation exposure.

Results: Of 303 children admitted during the study period, 159 underwent a total of 524 CXRs in PICU. Median (IQR) age of the study cohort was $2(0.6-5)$ years. More than two thirds $[n=115,72.3 \%]$ were mechanically ventilated. Most CXRs ( $n=449,85.7 \%)$ were performed on mechanically ventilated patients, amounting to a median (IQR) of $3(2-5)$ radiographs per ventilated patient. With increasing duration of ventilation, the number of CXRs proportionately increased in the first two weeks of mechanical ventilation. In non-ventilated children, about two thirds (68\%) underwent only one CXR. Majority of the prescriptions were on demand $(n=461,88 \%)$. Most common indications were peri-procedure prescriptions (37\%) followed by evaluation for respiratory disease status (24\%). About 40\% CXRs resulted in interventions; adjustment in ventilator settings (13.5\%) was the most frequent intervention. In 26\% $(n=138)$ of radiographs, image quality required improvement. One or more additional body part exposure other than chest and upper abdomen were noted 336 (64\%) images. Children with > 3 CXR had higher PRISM III score, more often mechanically ventilated, had higher number of indwelling devices [mean (SD) 2.6 (1.2) vs. 1.7 (1.0)] and stayed longer in PICU [median (IQR) 11(7.5-18.5) vs. 6 (3-9)].

Conclusion: On demand prescription was the prevalent practice in our PICU. Most non-ventilated children underwent only one CXR while duration of PICU stay and the number of devices determined the number of CXRs in mechanically ventilated children. Quality improvement strategies should concentrate on the process of acquisition of images and limiting the radiation exposure to unwanted body parts.

Keywords: PICU, Chest radiograph, X-ray, On demand, Quality improvement, Ventilation

*Correspondence: ny.karthi@gmail.com

1 Pediatric Emergency and Intensive Care Units, Department of Pediatrics, Advanced Pediatrics Centre, Postgraduate Institute of Medical Education and Research (PGIMER), Sector-12, Chandigarh 160012, India

Full list of author information is available at the end of the article

\section{Background}

Chest radiograph (CXR) is one of the most requested investigation in Intensive Care Units (ICU). Even in the era of modern non-radiation imaging techniques usefulness of a bedside portable CXR has remained unchanged 
[1]. With advent of digital radiography, images can be quickly captured, read, easily saved, retrieved, distributed and presented in a Picture Archiving Communication System [2]. Daily morning routine CXR prescription was the standard practice in ICU particularly in those receiving invasive mechanical ventilation (IMV), under the assumption that daily imaging can diagnose or identify undetected complications early [3]. However, recent evidence suggests that daily CXR in ICU patients receiving IMV are of low diagnostic yield and have negligible impact on management decisions and patient centered outcomes [4-8]. The Choosing Wisely campaign (2012) recommended not to use diagnostic tests including CXR on daily basis as they added no benefit to patient care besides increasing cost and potential harm [9-11]. Currently, though most intensivists prefer an on-demand strategy, a wide variation in practice has been reported across hospitals caring for adults and at some places, the daily routine CXR is still common [12-15].

Little is known about the CXR prescribing pattern and practice in Pediatric intensive care units (PICU). An important concern with respect to daily CXR, particularly in children is unwanted or avoidable radiation. For any given effective radiographic imaging, children get 3 to 4 times higher radiation dose as compared to adults; younger children get considerably more radiation in comparison to older counterparts [16]. In addition, frequent radiographic procedures could add to the risk of device (endotracheal tube, central venous catheters) displacement in understaffed units particularly common in a low middle income (LMIC) setting. Given this premise, we felt that a prospective evaluation of indications, prescribing patterns and clinical utility of portable chest radiographs performed in PICU could help in identifying potentially avoidable CXR and eventually pave way for quality improvement, cost savings and reduction in unwanted radiation in children.

\section{Methods}

This was a prospective observational study conducted in a tertiary PICU at a teaching and referral hospital from north India between December 2016 and April 2017. All children aged 12 years or younger admitted to PICU for whom a CXR was ordered were included. Children admitted to PICU in whom a CXR was performed outside the PICU premises [during Emergency Department (ED) stay or after surgical procedures in an X ray-suite] were excluded. Our PICU is an independent 15-bed medical unit, admitting about 900 children annually, and about half being mechanically ventilated. The consultants, pediatric intensive care fellows and postgraduates work in a twice daily rotation with availability of round the clock portable CXR facility. The investigator did not interfere with the imaging process, interpretation or any other aspect of clinical care. No additional imaging was performed as a part of this study. The study was approved by Institute Ethics Committee and a waiver of consent was granted.

\section{Data collection}

The study investigator (RG) prospectively collected information on all consecutive portable CXR prescribed from PICU during the study period. Data were recorded in a pre-designed proforma containing details regarding primary indication, clinical examination findings that prompted the request, prescribing physician's designation, timing of request, findings reported in CXR, quality and exposure of the images and interventions that were guided by CXR findings. Information regarding patient age, clinical diagnosis, type of respiratory support, duration of mechanical ventilation, number of indwelling devices and hospital outcomes were recorded.

\section{Study definitions}

The prescription strategy has been categorized as "routine" if CXR was performed in the morning hours between 5 and $8 \mathrm{AM}$ without specifying clinical indication in the request form. "On demand" was labelled if CXR was performed with a specific indication such as to confirm a positive clinical examination finding or verifying position of invasive lines/devices. High CXR exposure was defined when more than 3 CXRs were performed in a child during PICU admission.

\section{Outcomes}

The primary outcome was to assess the factors that were associated with higher CXR prescriptions during PICU stay. Secondary outcomes were to study the indications, nature of prescription, image quality and avoidable radiation exposure.

\section{Statistical analysis}

Descriptive statistics were summarized with mean (standard deviation) and median (interquartile range) for normal and skewed distribution respectively. Univariate statistical analysis was performed with t-tests (continuous variables), and Chi-square test or Fisher's exact test (categorical variables). Multivariable logistic regression analysis was carried out to identify the predictors of higher CXR prescription. All tests were two tailed and p-value less than 0.05 was taken as significant. Data analysis was done using SPSS statistical software (version 20.0, IBM SPSS Statistics, USA). 
Table 1 Characteristics of study subjects

\begin{tabular}{ll}
\hline Parameters & $\mathrm{n}=159$ \\
\hline Source of admission, n (\%) & \\
Emergency room & $137(86)$ \\
In-patient wards & $14(9)$ \\
Post-surgical & $8(5)$ \\
Duration of hospital stay before PICU admission in days, & $1(0-1)$ \\
Median (IQR) & \\
Age in months, Median (IQR) & $2(0.6-5)$ \\
Male, n (\%) & $84(52.8)$ \\
PRISM III score mean (SD) & $18.3(8.1)$ \\
Diagnosis n (\%) & \\
Pneumonia/ARDS & $32(20)$ \\
Empyema & $4(2.5)$ \\
Bronchiolitis/Bronchial asthma & $8(5)$ \\
Diffuse alveolar hemorrhage & $2(1.3)$ \\
Acute CNS infections (meningitis/encephalitis) & $23(14.5)$ \\
Status epilepticus & $6(3.8)$ \\
LGBS/myelitis/snake envenomation & $11(6.9)$ \\
Acute gastroenteritis & $5(3.1)$ \\
Scrub typhus/staphylococcal sepsis & $12(7.5)$ \\
Cardiac disorders (congenital/myocarditis) & $11(6.9)$ \\
Sepsis & $7(4.4)$ \\
Others & $38(24)$ \\
Respiratory support & \\
Invasive mechanical ventilation & $115(72.3)$ \\
Non-invasive ventilation & $02(1.3)$ \\
Non-ventilated & $42(26.4)$ \\
Outcome & \\
Survived to discharge & $117(73.6)$ \\
Discontinued care & $10(6.3)$ \\
Death & $32(20)$ \\
\hline
\end{tabular}

\section{Results}

Of 303 children admitted during the study period, 159 (52\%) who underwent at least one CXR while in PICU were enrolled into the study. Majority $(n=137,86 \%)$ were transferred from pediatric ED (Table 1). Median (IQR) age of the study population was $2(0.6-5)$ years. Nearly a third $(n=46,29 \%)$ were admitted with respiratory disorders; pneumonia and/or acute respiratory distress syndrome (ARDS), bronchiolitis and bronchial asthma were the most frequent diagnoses. Acute central nervous system (CNS) infections and status epilepticus were the second common group ( $\mathrm{N}=29,18.2 \%)$. Neuromuscular weakness, cardiac disorders and systemic infections/ sepsis comprised of about $7 \%$ of each. About three fourth of study subjects $(\mathrm{n}=115,72.3 \%)$ were mechanically ventilated and $2(1.3 \%)$ children received non-invasive ventilation. About a quarter had unfavorable outcome; thirty-two (20\%) children died and care was discontinued in another 10 (6\%) children.

During the study period of 116 calendar days, a total 524 CXRs were performed in 159 children, amounting to $4.5 \mathrm{CXR}$ per PICU day. Majority of the radiographs $(\mathrm{n}=449 ; 85.7 \%)$ were performed on mechanically ventilated patients. About one third (31\%) of all patients underwent only one CXR during their PICU stay. In nonventilated patients $(n=42,26.4 \%)$, this proportion rose to two thirds, i.e., about $68 \%$ of non-ventilated patients underwent only one CXR. However, in mechanically ventilated children, majority $(\mathrm{n}=75,65 \%)$ had 3 or more images [median (IQR)-3(2-5) radiographs per ventilated patient] (Table 2).

Most CXR prescriptions $(n=491,94 \%)$ were ordered by pediatric critical care fellows. (Table 3 ) Very few additional requests $(\mathrm{n}=14,2.5 \%)$ were made during morning

Table 2 Details of CXRs with respect to invasive ventilation

\begin{tabular}{llll}
\hline & Total $(\mathrm{n}=159)$ & Invasive ventilation $(\mathrm{n}=115)$ & $\begin{array}{c}\text { NIV \& Non- } \\
\text { ventilated } \\
(\mathrm{n}=44)\end{array}$ \\
\hline Total CXR performed n (\%) & & $449(85.7)$ & $75(14.3)$ \\
No. of CXR per PICU day Median (IQR) & 524 & & \\
No. of CXR per patient & $4.0(3-6)$ & $3.0(2-5)$ & $1.0(1-2)$ \\
Median (IQR) & $3.0(1-4)$ & $(1-18)$ & $(1-8)$ \\
Range & $(1-18)$ & $20(17.4)$ & $30(68.2)$ \\
No. of CXRs per patient n (\%) & & $20(17.4)$ & $7(16)$ \\
1 CXR & $50(31.4)$ & $22(19)$ & $3(6.8)$ \\
2 CXRs & $27(17)$ & $20(17.4)$ & $2(4.5)$ \\
3 CXRs & $25(15.7)$ & $12(10.4)$ & 0 \\
4 CXRs & $22(14)$ & $17(15)$ & $2(4.5)$ \\
5 CXRs & $12(7.5)$ & $4(3.5)$ & 0 \\
$6-10$ CXRs & $19(12)$ & $4(2.5)$ & \\
$>10$ CXRs & & \\
\hline
\end{tabular}


Table 3 Details of chest radiograph prescription and indications

\begin{tabular}{|c|c|}
\hline Characteristics & n (\%) \\
\hline CXR prescription ordered by & $n=524$ \\
\hline Consultant & $14(2.5)$ \\
\hline Fellow & $491(94)$ \\
\hline Postgraduate Resident & $11(2)$ \\
\hline Not specified & $08(1.5)$ \\
\hline Nature of prescription & $n=524$ \\
\hline Routine & $63(12)$ \\
\hline On demand & $461(88)$ \\
\hline Nature of indications in 'on-demand' prescriptions n (\%) & $n=461(88)$ \\
\hline Elective indications & $241(52)$ \\
\hline Urgent/emergent indications & $127(28)$ \\
\hline Unclassified & $93(20)$ \\
\hline Indications for CXR n (\%) & $n=524$ \\
\hline To evaluate the respiratory disease status & $125(23.8)$ \\
\hline Acute reduction in unilateral air entry & $86(16.2)$ \\
\hline New onset desaturation & $57(11)$ \\
\hline Peri-procedure & $194(37)$ \\
\hline Others & $62(12)$ \\
\hline Peri-procedure indications n (\%) & $n=194(37)$ \\
\hline Post-intubation & $79(41)$ \\
\hline Pre-extubation & $32(16.5)$ \\
\hline Post-extubation & $17(8.7)$ \\
\hline Central venous catheter position assessment & $14(7)$ \\
\hline Post intercostal tube insertion & $36(18.5)$ \\
\hline Post intercostal tube removal & $01(0.5)$ \\
\hline Confirmation of feeding tube position & 0 \\
\hline Post tracheostomy & $10(5)$ \\
\hline Post bronchoscopy & $05(2.5)$ \\
\hline Interventions after CXR n(\%) & $n=204(39)$ \\
\hline Endotracheal tube repositioning & $51(25)$ \\
\hline Central Venous Line repositioning & $02(1)$ \\
\hline Addition/change of antimicrobials & $04(2)$ \\
\hline Adjustment in Ventilator setting & $71(35)$ \\
\hline $\begin{array}{l}\text { Changes in supportive care (Physiotherapy, position } \\
\text { change) }\end{array}$ & $47(23)$ \\
\hline Intercostal tube drainage & $06(3)$ \\
\hline Others & $23(11)$ \\
\hline
\end{tabular}

rounds by a consultant. We observed a predominant on demand prescription $[n=461(88 \%)]$. Among the ondemand requests, about half had elective indications while just over a quarter $(\mathrm{n}=127,28 \%)$ had urgent or emergent indications for performing CXR as denoted in the request form. Although we intended to record the timeline of prescription to acquisition of image to identify any time delay in obtaining portable radiograph, the data was incomplete and hence not presented here. Peri-procedural prescriptions formed the most common indication for ordering a CXR $(n=194,37 \%)$, majority being after endotracheal intubation $(\mathrm{n}=79,15 \%)$, prior to extubation $(\mathrm{n}=32,6 \%)$ and post intercostal tube drainage $(n=36,7 \%)$. Only $2.5 \%(n=14)$ radiographs were performed for confirmation of position of central venous catheters and none were performed for confirming feeding tube position. The other frequent indications for a CXR were ascertaining the baseline pulmonary status ( $\mathrm{n}=125,23.8 \%)$, confirmation of abnormal clinical examination findings $(\mathrm{n}=86,16 \%)$ and hypoxemic episodes $(n=57,11 \%)$. About $40 \%$ of images resulted in interventions. Adjustment in ventilator setting was the most common intervention ( $\mathrm{n}=71,13.5 \%)$ followed by repositioning of endotracheal tube and changes in supportive care.

With increasing duration of invasive ventilation, the mean number of CXRs per patient proportionately increased in the first two weeks (Fig. 1). Children who were ventilated for 4 to 7 days duration underwent an average of 3.3 CXRs as compared to 5.9 CXRs in those who received mechanical ventilation for 8 to 14 days. However, in children requiring ventilation beyond 2 weeks, the average no. of CXRs had decreased to 5.1 images. Details about the process of acquisition of CXR images and interpretation are shown in Table 4. The image findings were obtained from clinical records and radiologist's reports and independently interpreted by investigators (RG \& KN) for agreement. Of 524 images, $15 \%(n=79)$ did not have any abnormal findings and were reported as normal. Unilateral/bilateral infiltrates, consolidation or collapse were the most common findings seen in $51 \%$ of images. Pneumothorax was noted in $6 \%$. In $26 \%(n=138)$ of radiographs, image quality required improvement. One or more additional body part exposure other than chest and upper abdomen were noted 336 (64\%) images.

\section{Multivariable analysis}

We compared children who underwent 3 or less CXRs with those who had $>3$ images during their PICU stay (Table 5). Children with $>3$ images had higher Pediatric risk of mortality III (PRISM III) score, more often mechanically ventilated, had higher number of indwelling devices [mean (SD) 2.6 (1.2) vs. 1.7(1.0)] and stayed longer in PICU [Median (IQR) 11(7.5-18.5) vs. 6 (3-9)]. On multivariable analysis, PRISM III score, length of PICU stay and number of indwelling devices were independently associated with higher CXR prescriptions.

\section{Discussion}

In this prospective study we found that most CXRs ordered in PICU were in mechanically ventilated children. The number of images increased proportionately during the first two weeks of invasive ventilation. Severity 


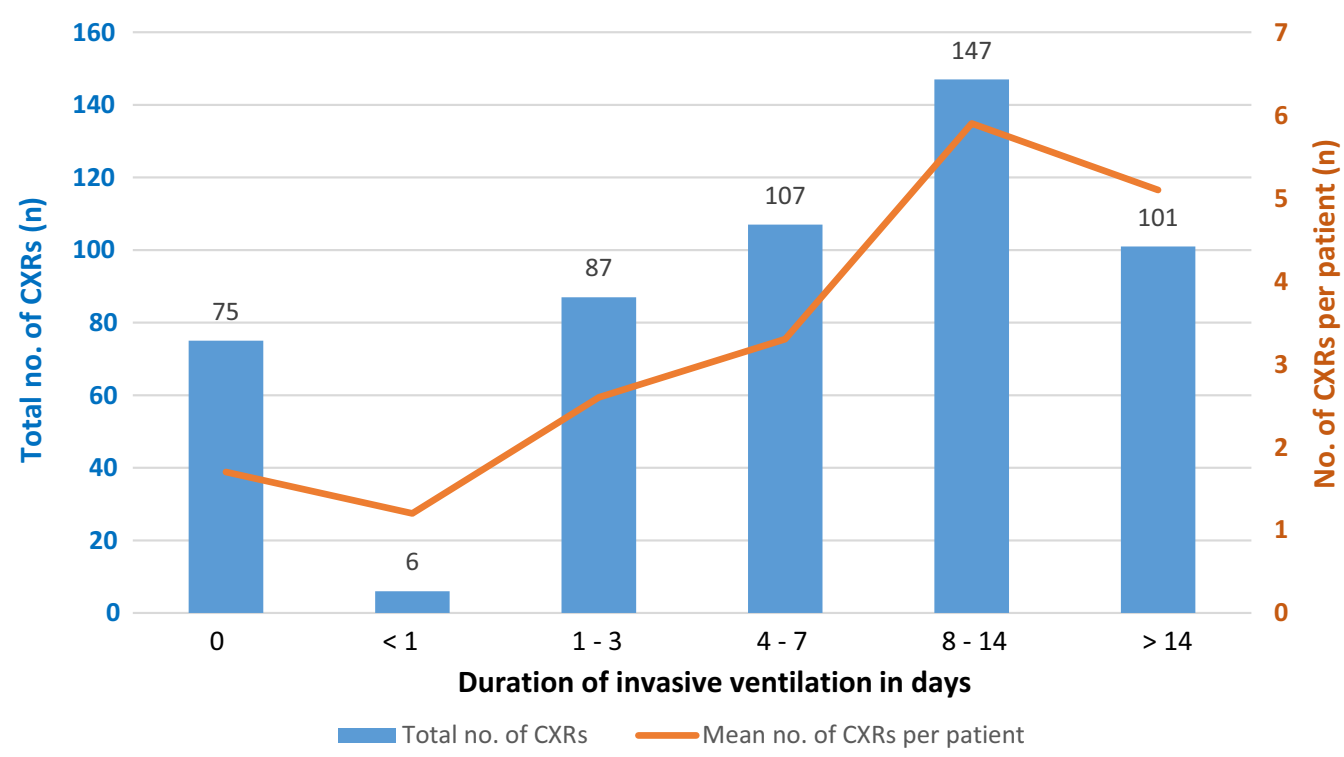

Fig. 1 Relationship between duration of invasive ventilation and number of CXRs

of illness at admission, number of indwelling devices and length of PICU stay were predictive of higher number of CXRs in children admitted to PICU. Only about half ( $\mathrm{n}=159,52 \%)$ of our PICU admissions underwent a chest radiograph imaging during PICU stay. This finding highlights two important dynamics in our PICU functioning. Firstly, all our study children were admitted in ED or ward for a median duration of about $24 \mathrm{~h}$ before transfer to PICU and it is likely that some of them had undergone a CXR immediately before PICU admission. Secondly, the prevalence of 'on-demand' prescription $(88 \%)$ could be another reason for the lower frequency of CXR prescription in admitted patients. Several centres have reported a change in practice from routine CXRs to on-demand prescriptions over the past two decades [1315] Although a prospective study conducted in multiinstitutional PICUs suggested routine CXRs to be useful in a cohort of critically ill children, subsequent randomized controlled trials in adults and consensus opinions strongly supported the adoption of on-demand strategy to minimize the use of radiography in mechanically ventilated patients without compromising quality of care or safety [3, 17].

Our study cohort included critically ill children who were younger and smaller; the median (IQR) age and weight were $2(0.6-5)$ years and $12.6(8.4) \mathrm{kg}$ respectively. This is similar to the report by Quasney et al., where the median weight of the study cohort was $9 \mathrm{~kg}$ (range $2-103 \mathrm{~kg}$ ) and 55\% of CXRs were performed in children weighing $\leq 10 \mathrm{~kg}$ [3]. This study also reported that the possibility of an intervention being performed based on routine CXR was higher in children weighing $\leq 10 \mathrm{~kg}$. Additionally, presence of indwelling devices was positively associated with CXR prescription and intervention, a finding similar to ours. Nearly $90 \%$ of routine CXRs were performed on children who had one or more devices and presence of 2 devices increased the intervention rate from $19 \%$ to $>50 \%$ [OR $(95 \% \mathrm{CI}) 5.3(2.5-11.5)$ ]. Of 524 CXRs performed in our study, most (85\%) were done on mechanically ventilated patients. This is in contrast to the previous study where only $65 \%$ were obtained in patients on mechanical ventilation [3]. This difference could have been due to the 'routine' prescription strategy practiced in the latter report. More than two thirds of non-ventilated children in our cohort required only one CXR while the number of CXR increased proportionately with duration of ventilation in children receiving mechanical ventilation. Children who required invasive ventilation for 1 to 3 days and 4 to 7 days were prescribed an average of 2.6 and 3.3 CXRs during their PICU stay, respectively. This figure compares favorably with the rate of prescription in a multicenter study where the mean (95\% CI) number of 'on demand' CXR per patient per day of mechanical ventilation was $0.75(0.6-0.8)$ [17]. Mechanically ventilated children, with presence of one or more devices, are at higher risk of respiratory complications and more likely to have dynamic changes in pulmonary findings. Hence, CXRs are frequently ordered in this cohort of PICU patients despite an 'on demand' approach.

We found that peri-procedure prescription (37\%) and evaluation of pulmonary status in respiratory diseases 
Table 4 Details on process of acquisition of chest radiographic images

\begin{tabular}{ll}
\hline & $N=524(\%)$ \\
\hline Details & \\
Adequate quality images & $334(64)$ \\
Incomplete penetration (requiring digital adjustment) & $52(10)$ \\
Image quality requiring improvement & $138(26)$ \\
$\quad$ Rotation & $97(18.5)$ \\
Artifact (Lines and tubes obstructing the field) & $17(3.2)$ \\
Inadequate inclusion/poor lung volume & $24(4.6)$ \\
Field of view & \\
Field of view limited to chest and upper abdomen & $188(36)$ \\
One or more additional fields exposed & $336(64)$ \\
Exposed fields & \\
Upper neck/lower face & $190(36.3)$ \\
Full face & $10(1.9)$ \\
Forearm—single & $17(3.2)$ \\
Forearm—both & $12(2.3)$ \\
Hand—single & $8(1.5)$ \\
Hand—both & $2(0.4)$ \\
Lower abdomen & $322(61)$ \\
Pelvis & $79(15)$ \\
Thigh—single & $3(0.6)$ \\
Thigh—both & $27(5.2)$ \\
Findings & \\
Normal & $79(15)$ \\
Not reportable & $33(6)$ \\
Interstitial infiltrates & $266(51)$ \\
Hyperinflation & $58(11)$ \\
Pneumothorax & $11(2)$ \\
Cardiomegaly with normal lung fields & $31(6)$ \\
\hline & $42(8)$ \\
& $4(0.7)$ \\
\hline
\end{tabular}

(17\%) were the most common on-demand indications for performing a CXR in our PICU. This is comparable to other studies where CXRs were often obtained after endotracheal intubation, intercostal tube placement and central venous catheterization and for diagnostic evaluation of pneumonia, ARDS and pneumothorax [15, 17-19]. The indications for a CXR after intubation and central line placement are not considered 'on-demand' and not supported by literature [15, 20,21]. Similarly, pre and post extubation radiographic images are not routinely recommended and need further review to decrease unnecessary images and radiation exposure. In our study, about $12 \%$ CXRs were performed on mechanically ventilated children without clear written indication prior to order. A careful study of these prescriptions could provide further opportunities to identify unnecessary $\mathrm{x}$-rays that can be avoided in PICU. However, such analysis can be difficult in intensive care settings. Studies show that intensivists tend to assume higher clinical value of CXR than its reported efficacy [18]. The ability of a negative CXR to exclude complications probably has a clinical impact that is often hard to study [19].

An intervention was performed post imaging in 204 out of 524 radiographs (39\%). Most frequent interventions included adjustments in the ventilator setting and institution of chest physical therapy. About 10\% of CXRs resulted in repositioning of devices such as endotracheal tubes and central venous catheters. The list of interventions after CXR in our study was comparable to previous report by Quasney et al. in their multi PICU study, although the proportions were different [3]. In mechanically ventilated children $\leq 10 \mathrm{~kg}$, about $20 \%$ of CXRs resulted in adjustment of ET tube while change in ventilator settings was performed after about $10 \%$ of images. Randomized trials in adults and in PICU did not demonstrate increased need of intervention in

Table 5 Predictors of higher CXR prescriptions

\begin{tabular}{|c|c|c|c|c|c|}
\hline \multirow[t]{2}{*}{ Variables } & \multirow[t]{2}{*}{$\leq 3$ CXRs $(n=102)$} & \multirow[t]{2}{*}{$>3$ CXRs $(n=57)$} & \multirow[t]{2}{*}{$p$ value } & \multicolumn{2}{|c|}{ Multivariable analysis } \\
\hline & & & & $P$ value & Odds ratio $(95 \% \mathrm{Cl})$ \\
\hline Age in years & $2(0.3-5.2)$ & $2(0.7-5)$ & 0.5 & & \\
\hline \multicolumn{6}{|l|}{ Median (IQR) } \\
\hline \multicolumn{6}{|l|}{ Diagnosis n (\%) } \\
\hline Respiratory diseases & $40(39)$ & $31(54)$ & 0.06 & & \\
\hline Neurological disorders & $34(33.3)$ & $16(28)$ & 0.49 & & \\
\hline PRISM III score mean (SD) & $16.5(7.6)$ & $21.6(8)$ & 0.001 & 0.02 & $1.07(1.0-1.1)$ \\
\hline Mechanically ventilated n (\%) & $62(61)$ & $53(93)$ & $<0.001$ & 0.15 & $2.8(0.7-11.7)$ \\
\hline No of indwelling devices mean (SD) & $1.7(1.0)$ & $2.6(1.2)$ & $<0.001$ & 0.006 & $1.9(1.2-3.0)$ \\
\hline Length of PICU stay median (IQR) & $6(3-9)$ & $11(7.5-18.5)$ & 0.001 & 0.03 & $1.04(1.0-1.1)$ \\
\hline
\end{tabular}


routine CXR group. Clec'h et al. in their randomised trial found restrictive use of CXRs in mechanically ventilated patients to have better diagnostic and therapeutic efficacies without affecting mortality [5] Similarly, another single centre randomised trial in PICU showed that daily routine CXRs was not associated with reduced length of stay or mortality [22]. In our cohort, the predominant indication for CXR was a respiratory cause while in most studies it was related to cardiovascular or postoperative cause.

Our results on details of acquisition of radiographs and quality of images provided some important insights into the process and identified areas for improvement. With the advent of portable digital CXR, rate of repeat radiographs came down significantly [23]. However, about a quarter of all images posed difficulty in interpretation of findings as they had axis rotation or presence of artifacts due to lines and tubes. Though portable CXRs in intensive care settings has its limitations, this process can be improved upon to avoid errors in interpretation and subjecting children to repeated radiation exposure. It was also noted that in about two thirds of CXRs, children had additional body part exposure other than chest and upper abdomen, a finding similar to previous reports [24]. It may be challenging to limit extra exposure in small children. An x-ray exposure chart was proposed by Knight et al. to optimize digital radiography in order to lower radiation dose and improve image quality $[25,26]$. However, the authors acknowledged the prevailing gaps in the literature in assisting the development of such a chart for children. The efforts require involvement of multiple stakeholders, including radiographers, radiologists, equipment vendors and clinicians. The exposures would need optimization based on age, size and equipment combination.

\section{Strengths and limitations}

Our study has several strengths. This is one of the first studies in PICU to report the prescription practice and the process of acquisition of images from LMIC setting. We measured the association between mechanical ventilation, number of devices and the prescription of CXRs in a sizeable sample. A few limitations need mention. We did not measure the alternative imaging modalities (eg. ultrasound) used during the study period that could potentially replace CXR in certain circumstances. An interventional study could have been a more useful methodology in quantifying potential areas of quality improvement.

\section{Conclusion}

On demand prescription for chest radiograph was the prevalent practice in our PICU. Most non-ventilated children underwent only one CXR while the length of PICU stay and the number of devices determined the number of CXRs in mechanically ventilated children. Quality improvement strategies should concentrate on the process of acquisition of images and limiting the radiation exposure to unwanted body parts.

\section{Abbreviations \\ CXR: Chest radiograph; ICU: Intensive care unit; IMV: Invasive mechanical ven- tilation; PICU: Pediatric intensive care unit; ED: Emergency department; LMIC: Low middle income countries; ARDS: Acute respiratory distress syndrome; CNS: Central nervous system; PRISM III: Pediatric risk of mortality III.}

\section{Acknowledgements}

Not applicable.

\section{Authors' contributions}

KN and RG conceptualized the study. All authors contributed to the formulation of study protocol. RG collected the data. RG, KN and VJ interpreted and analyzed the data. RG and KN wrote the initial draft. MJ and AKS provided critical inputs. RG, KN and VJ prepared the revised draft. All authors read and approved the final manuscript.

\section{Funding}

There is no funding/financial support involved in this study.

\section{Availability of data and materials}

All data generated or analysed during this study are included in this published article. The dataset/radiographic images are available from the corresponding author on reasonable request.

\section{Ethics approval and consent to participate}

The study was approved by Institute Ethics Committee, Postgraduate Institute of Medical Education and Research (Reference No. NK-3515/MD) and a waiver of consent was granted. All methods were performed in accordance with the relevant guidelines and regulations stated by the committee.

Consent for publication

Not applicable.

Competing interests

The authors declare that they have no competing interests.

\section{Author details \\ 1 Pediatric Emergency and Intensive Care Units, Department of Pediatrics, Advanced Pediatrics Centre, Postgraduate Institute of Medical Education and Research (PGIMER), Sector-12, Chandigarh 160012, India. ${ }^{2}$ Department of Radiodiagnosis, Postgraduate Institute of Medical Education and Research (PGIMER), Chandigarh, India.}

Received: 13 November 2020 Accepted: 26 February 2021

Published online: 09 March 2021

References

1. Eisenhuber E, Schaefer-Prokop CM, Prosch H, Schima W. Bedside chest radiography. Respir Care. 2012;57:427-43.

2. Schaefer-Prokop C, Uffmann M. Update on digital radiography. Eur J Radiol. 2009;72:193.

3. Quasney MW, Goodman DM, Billow M, Chiu H, Easterling L, Frankel L, et al. Routine chest radiographs in pediatric intensive care units. Pediatrics. 2001;107:241-8. 
4. Hendrikse KA, Gratama JWC, ten Hove W, Rommes JH, Schultz MJ, Spronk $P E$. Low value of routine chest radiographs in a mixed medical-surgical ICU. Chest. 2007;132:823-8.

5. Cle'ch C, Simon P, Hamdi A, Hamza L, Karoubi P, Fosse J-P, et al. Are daily routine chest radiographs useful in critically ill, mechanically ventilated patients? A randomized study. Intensive Care Med. 2008;34:264-70.

6. Krivopal M, Shlobin OA, Schwartzstein RM. Utility of daily routine portable chest radiographs in mechanically ventilated patients in the medical ICU. Chest. 2003:123:1607-14.

7. Ganapathy A, Adhikari NKJ, Spiegelman J, Scales DC. Routine chest x-rays in intensive care units: a systematic review and meta-analysis. Crit Care. 2012;16:R68.

8. Oba Y, Zaza T. Abandoning daily routine chest radiography in the intensive care unit: meta-analysis. Radiology. 2010;255:386-95.

9. Halpern SD, Becker D, Curtis JR, Fowler R, Hyzy R, Kaplan LJ, et al. An official American Thoracic Society/American Association of Critical-Care Nurses/American College of Chest Physicians/Society of Critical Care Medicine policy statement: the Choosing Wisely ${ }^{\circledR}$ Top 5 list in Critical Care Medicine. Am J Respir Crit Care Med. 2014;190:818-26.

10. Amorosa JK, Bramwit MP, Mohammed T-LH, Reddy GP, Brown K, Dyer DS, et al. ACR appropriateness criteria routine chest radiographs in intensive care unit patients. J Am Coll Radiol. 2013;10:170-4.

11. McComb BL, Chung JH, Crabtree TD, Heitkamp DE, lannettoni MD, ACR, , et al. Appropriateness criteria ${ }^{\circledR}$ routine chest radiography. Expert panel on thoracic imaging. J Thorac Imaging. 2016;31:W13-15.

12. Al Shahrani A, Al-Surimi K. Daily routine versus on-demand chest radiograph policy and practice in adult ICU patients-clinicians' perspective. BMC Med Imaging. 2018;18:4.

13. Gershengorn HB, Wunsch H, Scales DC, Rubenfeld GD. Trends in use of daily chest radiographs among US adults receiving mechanical ventilation. JAMA Netw Open. 2018;1:e181119.

14. Lakhal K, Serveaux-Delous M, Lefrant JY, Capdevila X, Jaber S, AzuRéa network for the RadioDay study group. Chest radiographs in 104 French ICUs: current prescription strategies and clinical value (the RadioDay study). Intensive Care Med. 2012;38:1787-99.

15. Tolsma M, Rijpstra TA, Schultz MJ, Mulder PG, van der Meer NJ. Significant changes in the practice of chest radiography in Dutch intensive care units: a web-based survey. Ann Intens Care. 2014;4:10.

16. Schaefer-Prokop C, Neitzel U, Venema HW, Uffmann M, Prokop M. Digital chest radiography: an update on modern technology, dose containment and control of image quality. Eur Radiol. 2008;18:1818-30.
17. Hejblum G, Chalumeau-Lemoine L, loos V, Boëlle P-Y, Salomon L, Simon T, et al. Comparison of routine and on-demand prescription of chest radiographs in mechanically ventilated adults: a multicentre, clusterrandomised, two-period crossover study. Lancet. 2009;374:1687-93.

18. Sy E, Luong M, Quon M, Kim Y, Sharifi S, Norena M, et al. Implementation of a quality improvement initiative to reduce daily chest radiographs in the intensive care unit. BMJ Qual Saf. 2016;25:379-85.

19. Graat ME, Hendrikse KA, Spronk PE, Korevaar JC, Stoker J, Schultz MJ. Chest radiography practice in critically ill patients: a postal survey in the Netherlands. BMC Med Imaging. 2006;6:8.

20. Lessnau K-D. Is chest radiography necessary after uncomplicated insertion of a triple-lumen catheter in the right internal jugular vein, using the anterior approach? Chest. 2005;127:220-3.

21. Lucey B, Varghese JC, Haslam P, Lee MJ. Routine chest radiographs after central line insertion: mandatory postprocedural evaluation or unnecessary waste of resources? Cardiovasc Intervent Radiol. 1999;22:381-4.

22. Bafaqih $H$, Almohaimeed $S$, Thabet F, Alhejaili A, Alarabi R, Zolaly M, Baqais K, Kasim K, Chehab M. Utility of daily routine portable chest X-ray in mechanically ventilated patients in the pediatric intensive care unit. J Pediatr Intensive Care. 2014;3(1):29-34.

23. Lotano R, Gerber D, Aseron C, Santarelli R, Pratter M. Utility of postintubation chest radiographs in the intensive care unit. Crit Care. 2000;4:50-3.

24. Bader D, Datz H, Bartal G, Juster AA, Marks K, Smolkin T, Zangen S, Kugelman A, Hoffmann C, Shani G, Ben-Shlomo A, Margaliot M, Sadetzki S. Unintentional exposure of neonates to conventional radiography in the Neonatal Intensive Care Units. J Perinatol. 2007;27:579-85.

25. Fintelmann F, Pulli B, Abedi-Tari F, Trombley M, Shore M-T, Shepard J-A et al. Repeat rates in digital chest radiography and strategies for improvement. J Thorac Imaging. 2012;27:148-51.

26. Knight SP. A paediatric X-ray exposure chart. J Med Radiat Sci. 2014;61:191-201

\section{Publisher's Note}

Springer Nature remains neutral with regard to jurisdictional claims in published maps and institutional affiliations.
Ready to submit your research? Choose BMC and benefit from:

- fast, convenient online submission

- thorough peer review by experienced researchers in your field

- rapid publication on acceptance

- support for research data, including large and complex data types

- gold Open Access which fosters wider collaboration and increased citations

- maximum visibility for your research: over $100 \mathrm{M}$ website views per year

At BMC, research is always in progress.

Learn more biomedcentral.com/submissions 